\title{
Drug Delivery Experiments in the ChE Curriculum
}

\author{
Stephanie Farrell, Robert P. Hesketh, Mariano J. Savelski, \\ and C. Stewart Slater \\ Department of Chemical Engineering \\ Rowan University
}

\begin{abstract}
Drug Delivery is a burgeoning field that represents one of the major research and development focus areas of pharmaceutical industry today, with new drug delivery system sales exceeding 10 billion dollars per year ${ }^{[1]}$. Chemical Engineers play an important and expanding role in this exciting field, yet undergraduate chemical engineering students are rarely exposed to drug delivery through their coursework. To provide students with the skills directly relevant to the evolving needs of the pharmaceutical industry, this project will develop and integrate applied drug delivery coursework and experiments throughout the Rowan Engineering curriculum.
\end{abstract}

To design and produce a new drug delivery system, an engineer must fully understand the drug and material properties and the processing variables that affect the release of the drug from the system. This requires a solid grasp of the fundamentals of mass transfer, reaction kinetics, thermodynamics and transport phenomena. The engineer must also be skilled in characterization techniques and physical property testing of the delivery system, and practiced in the analysis of the drug release data.

This project aims to provide engineering students with skills relevant to the field of drug delivery. This paper describes seven modules in which students apply engineering principles to the design, preparation, characterization, and analysis of drug delivery systems. A variety of drug delivery systems are explored including tablets, transdermal delivery systems, osmotic pumps, and supercritical fluid-processed particles. Experiments were developed to investigate the rate controlling mechanisms of different types of controlled release systems and to explore drug stability and to determine shelf life.

Introduction

This project comprises seven modules that introduce students to multidisciplinary engineering principles through application to drug delivery systems. This project modifies measurement techniques and laboratory experiments widely used in the pharmaceutical sciences, to teach engineering principles. Material from the seven modules is being integrated vertically into the curriculum beginning with the Freshman Clinic, then fundamental Engineering courses, followed by Junior-Senior Clinic research projects, and finally advanced level electives on pharmaceutical topics. At the freshman level, students are engaged in the scientific discovery process with exciting hands-on analysis of commercial drug delivery systems. In more advanced courses, students design and formulate drug delivery systems and investigate the variables affecting their behavior. The Junior/Senior Clinic provides an opportunity for students to perform research projects related to drug delivery in a multidisciplinary setting. A 
senior/graduate level elective course, "Drug Delivery: Theory and Applications" has also been been developed.

Controlled drug delivery systems attempt to deliver a drug to the body at a controlled rate for an extended period of time. Historically, drug delivery systems were developed primarily for traditional routes of administration such as oral and intravenous. Recently, however, there has been an explosion in research on delivery by so-called non-conventional routes, such as transdermal, nasal, ocular, and pulmonary administration. Drug delivery applications have expanded from traditional drugs to therapeutic peptides, vaccines, hormones, and viral vectors for gene therapy. These systems employ a variety of rate-controlling mechanisms, including matrix diffusion, membrane diffusion, biodegradation and osmosis. To design and produce a new drug delivery system, an engineer must fully understand the drug and material properties and the processing variables that affect the release of the drug from the system. This requires a solid grasp of the fundamentals of mass transfer, reaction kinetics, thermodynamics and transport phenomena. He or she must also be skilled in characterization techniques and physical property testing of the delivery system, and practiced in the analysis of the drug release data.

The engineering goals of this project are (1) to explore different types of drug delivery systems; (2) to study drug delivery designs in a quantitative manner using engineering principles; (3) to use up-to-date industrial techniques for the production, testing and analysis of drug delivery systems; and (4) to evaluate factors influencing the drug release from a delivery system.

Module \#1: Drug release from a solid tablet

Oral ingestion has long been the most convenient and commonly used route of drug delivery. For this reason, design and manufacture of oral formulations such as tablets and capsules is a very important aspect of drug delivery in the pharmaceutical industry. To prepare a sustained release tablet of a water-soluble drug, the drug is mixed with a hydrophobic matrix, and compressed into tablet form using a standard method such as the direct compression method or dry granulation method. Drug, matrix, and process parameters affect the tablet's physical properties which include hardness, disintegration and dissolution. These properties can be

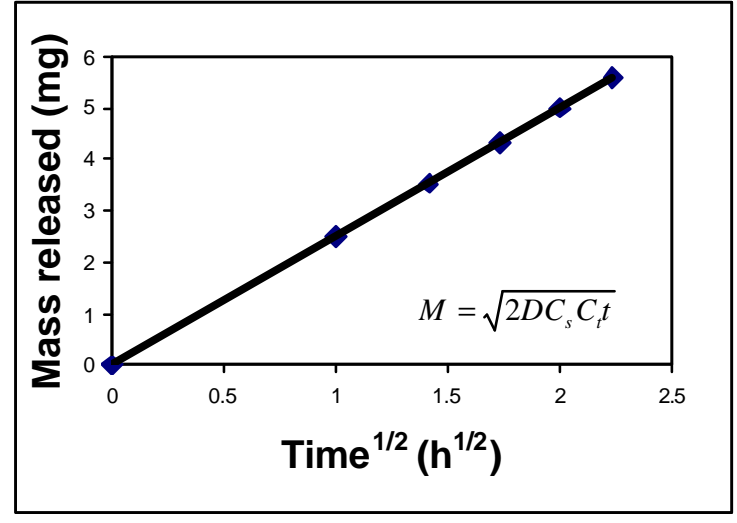

Figure 1. Higuchi drug release follows a square-root of time dependence. evaluated using standard methods commonly taught in Pharmaceutical Science courses and widely used by scientists and engineers in the pharmaceutical industry. Drug release kinetics from tablet matrices commonly follow a squareroot-of-time dependence first described by Higuchi ${ }^{[2]}$ as shown in Figure 1.

In this module, students produce drug matrices in tablet form for caffeine delivery. The objectives of this module are to (1) introduce students to the advantages and disadvantages of tablet dosage forms; (2) prepare tablet dosage forms, (3) investigate how drug concentration, polymer concentration, and polymer molecular weight effect a tablet's physical properties ${ }^{[3]}$, (4) investigate the release kinetics of the drug from the matrix and to determine whether Higuchi 
kinetics ${ }^{[2]}$ are followed. Drug release profiles are determined by analysis of drug concentration using UV spectrophotometry.

Students prepare several different tablet formulations as shown in Table 1. POLYOX N-10 and $\mathrm{N}$-303 differ in molecular weight.. Caffiene is the active ingredient (drug), and POLYOX is the polymeric gel matrix material which controls the release rate. Lactose is a commonly-used binder/filler, and magnesium stearate is often used as a powder lubricant in tablet formulation. The effect of drug concentration is shown in Figure 2, and the effect of polymer concentration is shown in Figure 3

Table 1: Tablet Formulations

\begin{tabular}{lccccc}
\hline Ingredients & A & B & C & D & E \\
\hline \hline Caffeine $(\mathrm{g})$ & 0.12 & 0.24 & 0.36 & 0.24 & 0.24 \\
\hline $\begin{array}{l}\text { POLYOX (g) (N-303 or N- } \\
\text { 10) }\end{array}$ & 0.24 & 0.24 & 0.24 & 0.12 & 0.72 \\
\hline Lactose (g) & 0.828 & 0.708 & 0.588 & 0.828 & 0.228 \\
\hline Magnesium Stearate $(\mathrm{g})$ & 0.012 & 0.012 & 0.012 & 0.012 & 0.012 \\
\hline
\end{tabular}

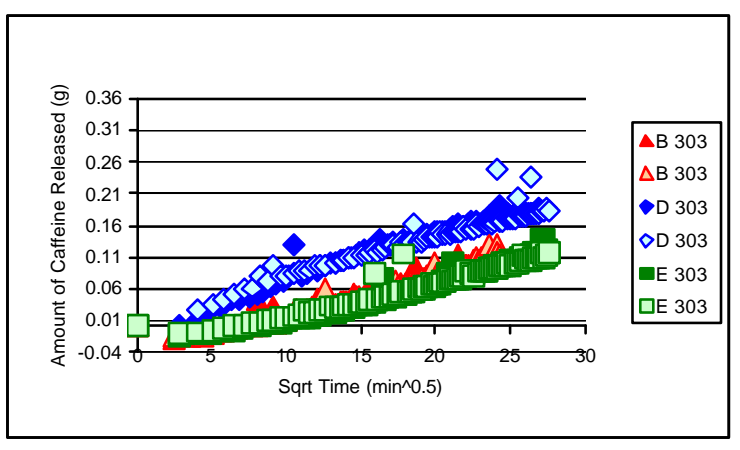

Figure 2. Effect of polymer concentration on drug release rate. Tablets used were formulations B, D, and E.

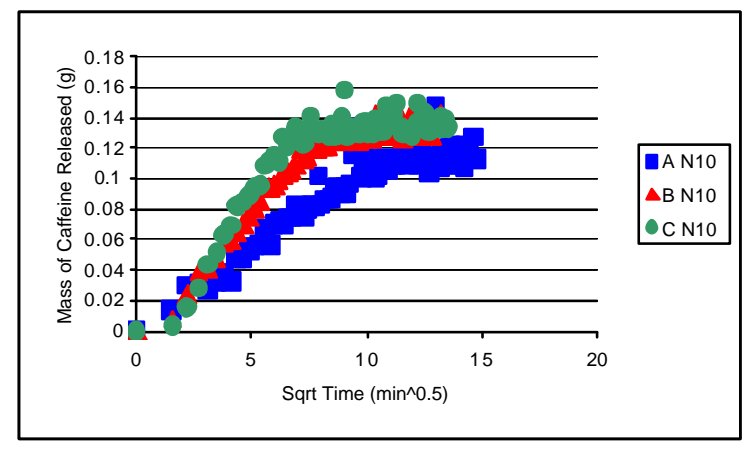

Figure 3. Effect of drug concentration on drug release rate from POLYOX N-10 tablets. Tablets used were formulations $\mathrm{A}, \mathrm{B}$, and $\mathrm{C}$. 
Module \#2: Drug release from a membrane system

Membrane-based

drug delivery devices are

another commonly used

system available in a variety

of forms such as microbeads,

transdermal patches and oral

formulations. In a membrane

system, the drug is contained

in a reservoir which is

surrounded by a coating

(membrane) that controls the rate of release of the drug.

Membrane systems are

capable of achieving a constant rate of drug delivery for an extended time ${ }^{[4,5,6,7]}$. One of the first membrane systems for controlled release was the Transderm-Scop ${ }^{\circledR}$ patch developed by Ciba (Woodbridge, NJ) for the control of motion sickness (Figure 4) ${ }^{[8]}$. Alza Corp (Palo Alto, CA) developed the Ocusert ${ }^{\circledR}$ system for 7-day ocular delivery of pilocarpine in the treatment of glaucoma $^{[9,10]}$. Transdermal nicotine delivery patches are now available, by prescription and ovr-the-counter, to assist with smoking cessation.

The release rate from a membrane device is related to the drug concentration in the reservoir $\left(\mathrm{C}_{\mathrm{r}}\right)$, and the permeabilities $(\mathrm{P})$ in the different layers:

$$
\frac{d M}{d t}=\frac{A C_{r}}{\frac{1}{P_{1}}+\frac{1}{P_{2}}}
$$

In this module, students explore how a membrane-controlled nicotine delivery system works by making their own patch. The patch is similar in construction to the commercial patches that deliver nicotine for a period of 16 hours, but it is designed to deliver most of the nicotine within a 2-hour laboratory timeframe. The patch contains three layers: A backing layer, a reservoir layer, and a rate-controlling membrane. The backing layer was a heat-sealable, polyester film laminate (3M, Minneapolis, MN). The reservoir layer was a $0.65 \mu \mathrm{m}$ porous hydrophilic PVDF Durapore membrane (Millipore, Bedford, MA), and the ratecontrolling membrane was a heat-sealable, hydrophilic, EVA (9\% vinyl acetate) membrane (3M) The reservoir layer was loaded with $20 \mu \mathrm{L}$ of nicotine before being heat-sealed between

Figure 5 Nicotine release from the EVA ratecontrolling membrane system. 
the backing and EVA membrane layers. The release experiment was performed by placing the patch in a beaker containing $80 \mathrm{ml}$ water, stirred continuously. Samples were taken at ten minute intervals and analyzed spectrophotometrically to determine the nicotine concentration. The results of the nicotine patch drug release experiment are shown in Figure 5.

Module \#3: Ointments: Preparation and evaluation of drug release

Ointments are used for topical delivery of agents such as antiseptics, antibiotics, and corticosteroids. Release of drugs from ointment bases occurs by diffusion from a matrix type system. The kinetics of drug release follow the Higuchi square-root of time dependence ${ }^{[2]}$.

In this module, students will prepare their own ointment formulations containing salycilic acid, and will evaluate the drug release kinetics from this system. The objectives are (1) to investigate the variables that affect the release rate of a drug from an ointment: the type of ointment base, the drug solubility in the base, and the drug concentration, (2) to perform drug release studies on the drug from the ointment, (3) to investigate the release kinetics of the drug from the ointment matrix and to determine whether Higuchi kinetics ${ }^{[2]}$ are followed.

Module \#4: Drug delivery using an osmotic pump

The osmotic pump developed by Alza exploits

osmosis to achieve a constant release rate of drug for an extended time. This technology has been applied to implant systems for delivery of many drugs for treatment of diseases such as Parkinson's and Alzheimer's, cancer, diabetes, and cardiovascular disorders. Efidac ${ }^{\circledR} 24$ hour nasal decongestants are an example of an oral system that uses the same technology.

The osmotic pump comprises three concentric layers: an innermost drug reservoir contained within an impermeable membrane, an osmotic solution, and a rigid outer layer of a

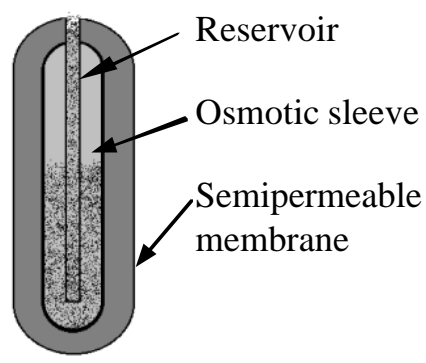
rate-controlling semi-permeable membrane (Figure 6). As water from the body permeates through the outermost membrane and into the osmotic "sleeve", the sleeve expands and compresses the innermost drug reservoir. This squeezes the drug out of the reservoir through a delivery portal ${ }^{[11]}$. The rate of drug release is proportional to the rate at which water flows into the "osmotic sleeve" due to an osmotic imbalance $\Delta \pi^{[12]}$ :

$$
\frac{d V}{d t}=\frac{A k}{h}(\Delta \pi)
$$

where $\mathrm{A}, \mathrm{k}$ and $\mathrm{h}$ are the membrane area, permeability and thickness, respectively.

In this module students will fill pre-made osmotic pump devices with a drug (caffeine solution), and will measure the rate of release of the drug from the device. The objectives of the experiments are (1) to investigate the release rate of drug from the device and to compare the release rate to the manufacturers specifications, (2) to compare the release profile to that predicted by the mathematical model described above and (3) to study the effects of temperature and osmolality on the release rate of the drug, and to compare with the model given by Theeuwes [11].

Proceedings of the 2005 American Society for Engineering Education Annual Conference and Exposition Copyright (C) 2005, American Society for Engineering Education 


$$
Q_{t}=Q_{0}\left(0.135 e^{(0.054 T)}-(.0 .004 \pi)+0.3\right)
$$

where $\mathrm{Q}_{\mathrm{t}}$ and $\mathrm{Q}_{0}$ are pumping rates at temperature $\mathrm{T}$ and $37^{\circ} \mathrm{C}$ respectively. Freshman students will perform a simple experiment to analyze a commercial oral osmotic system such as Efidac ${ }^{\circ}$.

Module \#5: Microcapsules: preparation and evaluation of drug release

Microencapsulation is one of the most intriguing fields in the area of drug delivery. It is an interdisciplinary field that requires knowledge of the field of polymer science, familiarity with emulsion technology, and an understanding of drug and protein behavior ${ }^{[13]}$. Testing of microcapsule release rates requires knowledge of the behavior and modeling of membrane diffusion systems. Pharmaceutical applications of microencapsulation technology include theophylline, heparin, anti-tumor drugs, gene therapy vectors, and vitamins, and current research is being done on such exciting applications as artificial red blood cells, and for treatment of acute kidney failure and other life-threatening conditions ${ }^{[14]}$.

In this module students will prepare microcapsules containing theophylline, a drug used in the treatment of asthma, and they will study the release rate of drug into simulated gastric fluid. The objectives are (1) to prepare theophylline-containing microcapsules of water-insoluble whey based protein, (2) to study the effect of microcapsule size, type of simulated digestive fluid, and extent of cross linking on the drug release (3) to determine whether the system is membranecontrolled or matrix diffusion-controlled by comparing the release profile to the appropriate models (the system is matrix diffusion controlled and follows Higuchi kinetics ${ }^{[15]}$.

Module \#6: Chemical kinetics: Drug stability

The chemical stability of a drug in a dosage form is of great interest since a drug may become therapeutically ineffective as it degrades. Additionally, drug decomposition may yield toxic by-products that are harmful to the patient.

In this module students test the stability of aspirin, which undergoes hydrolysis to form products of salicylic acid and acetic acid. Aspirin hydrolysis is a second order reaction, but in buffered solution follows apparent first-order kinetics ${ }^{[16]}$. The rate expression may be written as:

$$
\frac{-d A}{d t}=K_{a p p} A
$$

where,

$$
\begin{aligned}
\mathrm{A} & =\text { mass of aspirin }(\mathrm{mg}) \\
\mathrm{K}_{\text {app }} & =\text { a constant equal to } \mathrm{K}\left[\mathrm{OH}^{-}\right] \text {, with units of } \min ^{-1}(\mathrm{~K} \text { is the original second order } \\
& \text { rate constant, and [OH-] is the concentration of hydroxyl ions. }
\end{aligned}
$$

The integrated form of this rate expression is:

$$
\ln A_{t}=\ln A_{0}-K_{a p p} t
$$

where, 


$$
\begin{array}{ll}
\mathrm{A}_{\mathrm{t}} \quad \begin{array}{l}
=\text { mass of aspirin remaining at } \\
\text { time } \mathrm{t}(\mathrm{mg})
\end{array} \\
\mathrm{A}_{0} \quad \begin{array}{l}
=\text { mass of aspirin initially } \\
\text { present }(\mathrm{mg})
\end{array} \\
\mathrm{K}_{\text {app }} & \begin{array}{l}
\text { apparent first order rate } \\
\text { constant }\left(\mathrm{min}^{-1}\right)
\end{array} \\
\mathrm{t} \quad & =\text { time of sampling (min). }
\end{array}
$$

The rate constant $\mathrm{K}_{\mathrm{app}}$ can be determined from a plot of $\ln \left(\mathrm{A}_{\mathrm{rem}}\right)$ vs. time as shown in Figure 7. In order to determine the shelf life of the drug at room temperature, it is necessary to determine the temperature dependence of the apparent rate constant, which exhibits an Arrhenius dependence:

$$
\ln K_{a p p}=\ln \alpha-\frac{E_{a}}{R} \cdot \frac{1}{T}
$$

$$
\begin{array}{ll}
\mathrm{a} & =\text { frequency constant }\left(\mathrm{min}^{-1}\right) \\
\mathrm{E}_{\mathrm{a}} & =\text { activation energy }(\mathrm{cal} / \mathrm{mol}) \\
\mathrm{R} & =\text { gas constant }(1.987 \mathrm{cal} . / \mathrm{K} \mathrm{mol}) \\
\mathrm{T} & =\text { absolute temperature }(\mathrm{K})
\end{array}
$$

The shelf life at room temperature, t90 $_{0}$, is defined as the time for which $90 \%$ of the original aspirin remains (at room temperature, $298 \mathrm{~K}$ ):

$$
\ln (0.9)=-K_{a p p} t_{90}
$$

The objectives of this module are to (1) use accelerated stability testing at elevated temperatures to predict the shelf life of the drug room temperature $\left(25^{\circ} \mathrm{C}\right)$. (2) to investigate temperature and $\mathrm{pH}$ dependence of aspirin degradation (3) to distinguish between zero, first and second order reactions and rates (4) to use drug degradation data to construct an Arrhenius plot and determine activation energy, first order rate constant at room temperature, and shelf life at room temperature.

\section{Module \#7: Supercritical Fluid Technology in Drug Delivery}

Supercritical fluid technology (SFT) using environmentally benign agents such as $\mathrm{CO}_{2}$ is an emerging technology in the field of drug delivery. SFT has been used to prepare drug delivery systems of various types: polymeric particles, plain drug particles, drug-containing liposomes, and inclusion complexes of drug and carrier. In comparison with traditional techniques for preparation of these types of systems, SFT enables more control over formulation, thereby allowing more precise control of drug release from delivery systems ${ }^{[17]}$.

Proceedings of the 2005 American Society for Engineering Education Annual Conference and Exposition Copyright (C) 2005, American Society for Engineering Education 
According to Kompella ${ }^{[17]}$, "scant literature is available on the solubility of drugs in supercritical carbon dioxide". In this module, students will determine the solubility of a drug in $\mathrm{CO}_{2}$. and will also use a supercritical fluid process to obtain plain drug particles. In determining the drug solubility in supercritical $\mathrm{CO}_{2}$ a phase monitor will be used for direct visual observation of the supercritical fluid solution, and to ensure there is no liquid phase present. The objectives of this module are (1) to determine the solubility of a drug in supercritical $\mathrm{CO}_{2}$ and (2) to inve stigate the effect of SFT process variables such as flow rate, temperature and pressure on the mean drug particle size.

\section{Equipment}

Since one of the goals of this project is to provide students with background and training that would enhance their preparation for careers in the pharmaceutical industry, hands-on experience with modern drug delivery production and testing equipment is essential. The existing laboratory facilities include relevant analytical instrumentation (HPLC and spectrophotometer, both Hewlett Packard), and a supercritical extraction unit (Supercritical Fluid Technologies, Newark, DE). To implement this project, the following equipment will be purchased: a supercritical fluid phase monitor system, an in-line automated drug delivery system, and a tablet press. Provided below is more detailed information on the equipment being considered.

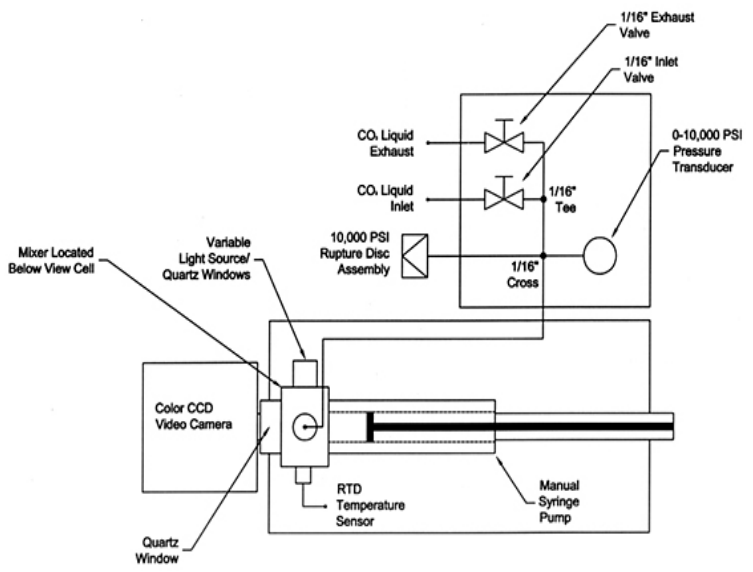

Figure 8. Schematic representation of the SFT Phase Monitor System. [SFT promotional literature]

Supercritical Fluid Phase Monitor System (Supercritical Fluid Technologies, Newark, DE). $(\$ 16,000)$ : This system facilitates drug solubility studies and direct visualization of drug particle formation in the supercritical fluid experiments. The Phase Monitor system is shown in Figure 8 .

In-Line Automated Drug Delivery System (Model ILC-14, PermeGear, Riegelsville, PA) $(\$ 22,250)$ : This is an automated system for drug release studies, necessary for experiments that extend beyond the laboratory period. This system is to be integrated with the existing spectrophotometer, to allow sampling and analysis for extended-duration experiments This system will be used in Modules 1-6 for analysis of drug release from extended release tablets, osmotic systems, membrane systems, ointments, and microspheres. The automated drug delivery system is shown in Figure 9.

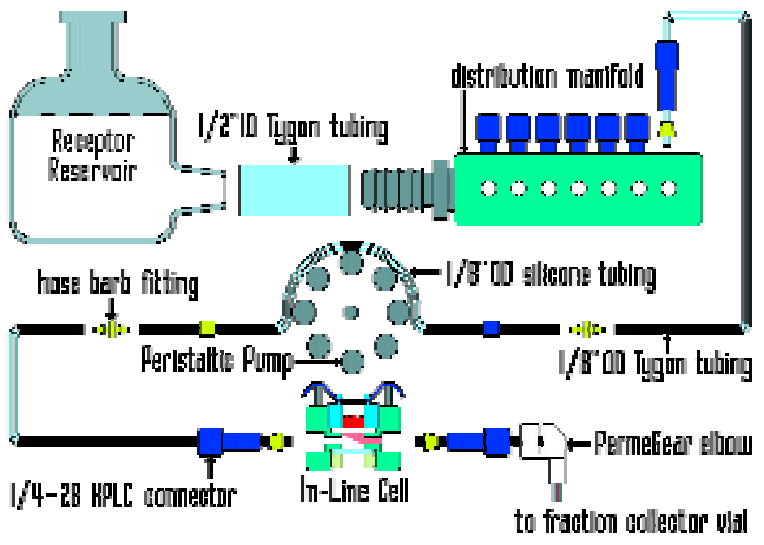

Figure 9. PermeGear ILC14 Automated Drug Delivery System. [Permegear promotional literature] 
A manual twelve ton singletablet press (Carver, Wabash, IN) was purchased for the solid tablet production module. This small bench-top machine is designed for pressing round tablets from various granular materials, applicable to laboratory used for research and development. Only one set of punch and die is mounted, leaving the depth of the filling material and the thickness of the tablets adjustable. This equipment is standard for tablet preparation on a small scale, and will be used in module \#1 on tablet investigation. The tablet press is shown in Figure 10.

\section{Summary}

Chemical Engineers play an important and expanding role the field of drug delivery, yet undergraduate chemical engineering students are rarely exposed to drug delivery through their coursework. To provide students with the skills directly relevant to the evolving needs of the pharmaceutical industry, we are developing and integrating applied drug delivery coursework and experiments throughout the Rowan Engineering curriculum.

Through several modules, integrated from freshman through senior and graduate level courses, students learn how fundamental engineering principles are applied in the design and production of drug delivery systems. They discover how the drug and material properties and the processing variables affect the release of a drug from a system. They acquire hands-on experience with characterization techniques and physical property testing of the delivery system, and become practiced in

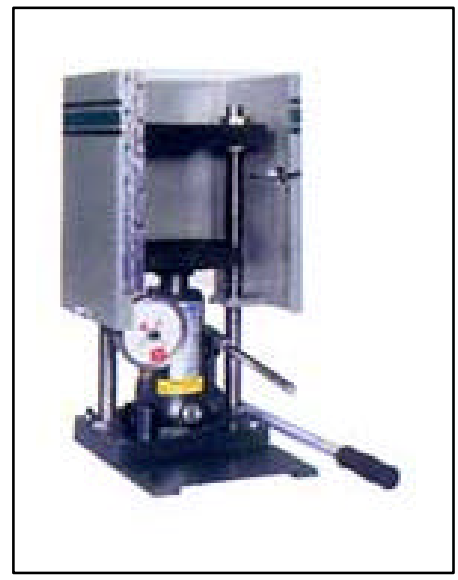

Figure 10. The Carver Mini-C Tablet Press the analysis of the drug release data. Students gain experience with modern industrial techniques for the production, testing, and analysis of drug delivery systems. Through the seven modules, a variety of drug delivery systems are explored: tablets, ointments, membrane systems, microcapsules, osmotic pumps, and supercritical fluid-processed particles.

\section{Acknowledgment}

This work was funded through a grant from the National Science Foundation's Course, Curriculum and Laboratory Improvement Program under grant DUE-0126902. Additional support was provided through the NSF REU Program, Grant \# 0353744. We gratefully acknowledge 3M Drug Delivery for their donation of the backing and EVA membrane materials, and Millipore Corporation for their donation of the Durapore membranes.

\section{References}

1 Langer, R., Foreward to Encyclopedia of Controlled Drug Delivery, Volume 1, Edith Mathiowitz (ed.), John Wiley and Sons, NY 1999.

2 Higuchi, T., "Rate of release of medicaments from ointment bases containing drugs in suspension", J. Pharm. Sci., 50 (10), p. 874-5, 1961.

3 Desai, S.J., et al., "Investigation of factors influencing release of solid drug dispersed in inert matrices", J. Pharm. Sci. 54(10), 1965. 
4 Flynn, G.L., S.H. Yalkowsky and T.J. Roseman, "Mass Transport Phenomena and Models: Theoretical Concepts”, J. Pharm. Sci., 63(4), 479-510, 1974.

5 Baker, R. and H. Lonsdale, "Controlled Drug Delivery- an emerging use for membranes", Chemtech, 667-674, Nov. 1975.

6 Tojo, K., Y. Sun, M.M. Ghannam and Y.W. Chien, "Characterization of a membrane permeation system for controlled drug delivery studies", AIChE J., 31(5), 741-746, 1985.

7 Chien, Y.W., "Transdermal Therapeutic Systems", in Controlled Drug Delivery Fundamentals and Applications, $2^{\text {nd }}$ ed., J. Robinson and V. Lee (eds.), Marcel Dekker, NY, 1987.

8 Shaw, J.E. and J. Urquhart, Trends Pharmacol. Ther, 29, 414-419, 1981.

9 Sendelbeck, L., D. Moore and J. Urquhart, Am. J. Ophthamol. 80, 274-283, 1975.

10 U.S. Patent 4,014,335 (March 29, 1977), R.K. Arnold (to Alza Corp.).

11 Theeuwes, F. and SI Yum, "Principles of the design and operation of generic osmotic pumps for the delivery of semisolid or liquid drug formulations", Ann Biomed Eng, 4(4), p. 343-353, 1976.

12 Hui, H, J. Robinson, and V. Lee, "Design and Fabrication of Oral Controlled Release Drug Delivery Systems" in Controlled Drug Delivery Fundamentals and Applications, $2^{\text {nd }}$ ed., J. Robinson and V. Lee (eds.), Marcel Dekker, NY, 1987.

13 Mathiowitz, E., Encyclopedia of Drug Delivery, Volume 2. John Wiley and Sons, NY, 1999.

14 Physician's Desk Reference, Medical Economics Data Production Company, Montvale, NJ, 1994, pp. 23852388.

15 Lee, S.J., and M. Rosenberg, "Preparation and properties of glutaraldehyde cross-linked whey protein-based microcapsules containing theophylline", J. Control Rel. 61, 123-136, 1999.

16 Remington: The Science and Practice of Pharmacy, $19^{\text {th }}$ ed., Mack Publishers, 1995.

17 Kompella, U.B. and K. Koushik, "Preparation of drug delivery systems using supercritical fluid technology", Critical Reviews in Therapeutic Drug Carrier Systems, 18(2), 173-199, 2001.

Biographical Information

STEPHANIE FARRELL is Associate Professor of Chemical Engineering at Rowan University. She received her B.S. from the University of Pennsylvania, her MS from Stevens Institute of Technology, and her Ph.D. from New Jersey Institute of Technology. Prior to joining Rowan in September, 1998, she was a faculty member in Chemical Engineering at Louisiana Tech University. Stephanie has research expertise in the field of drug delivery and controlled release, and she is currently focusing efforts on developing laboratory experiments related to membrane separations, biochemical engineering, and biomedical systems .

ROBERT HESKETH is Associate Professor of Chemical Engineering at Rowan University. He received his B.S. from the University of Illinois and his Ph.D. from the University of Delaware. After his Ph.D. he conducted research at the University of Cambridge, England, and joined the faculty at the University of Tulsa in 1996. Robert employs innovative methods such as cooperative learning and inductive teaching techniques in his classes

Proceedings of the 2005 American Society for Engineering Education Annual Conference and Exposition Copyright (C) 2005, American Society for Engineering Education 
MARIANO J SAVELSKI is Assistant Professor of Chemical Engineering at Rowan University. He received his B.S. in 1991 from the University of Buenos Aires, his ME in 1994 from the University of Tulsa and his Ph.D. in 1999 from the University of Oklahoma. His technical research is in the area of process design and optimization with over seven years of industrial experience. His prior academic experience includes two years as Assistant Professor in the Mathematics Department at the University of Buenos Aires.

C. STEWART SLATER is Professor and Chair of the Department of Chemical Engineering at Rowan University. He received his B.S., M.S. and Ph.D. from Rutgers University. Prior to joining Rowan, he was Professor of Chemical Engineering at Manhattan College. Dr. Slater's research and teaching interests are in separation and purification technology, laboratory development, and investigating novel processes for fields such as bio/pharmaceutical/food engineering and specialty chemical manufacture. 